\title{
Performance Analysis of a Non-identical Unit System with Priority and Weibull Repair and Failure Laws
}

\author{
Kuntal Devi \\ Research Scholar \\ Department of Maths. \& Stats. \\ Manipal University Jaipur \\ Jaipur-303007
}

\author{
Ashish Kumar \\ Assistant Professor \\ Department of Maths. \& Stats. \\ Manipal University Jaipur \\ Jaipur-303007
}

\author{
Monika Saini \\ Assistant Professor \\ Department of Maths. \& Stats. \\ Manipal University Jaipur \\ Jaipur-303007
}

\begin{abstract}
The objective of the present paper is to analyze the performance of a two non-identical unit system by considering Weibull distributed random variables. The concept of priority to preventive maintenance of original unit over repair of duplicate unit is also used. A single repairman is available for doing all repair activities. Preventive maintenance of the unit after a pre-specific time to enhance the performance and efficiency of the system conduct by repairman. Recurrence relations for various measures of system effectiveness are derived by using semi-Markov process and regenerative point technique. The system is observed at numerical results for MTSF, steady state availability and profit function has derived for particular case.
\end{abstract}

\section{Keywords}

Non-identical Units; Weibull Failure and Repair Laws; Preventive Maintenance; Priority and Maximum Operation Time

\section{INTRODUCTION}

Many researchers like, Cao and $\mathrm{Wu}$ [2], Agnihotri and Satsangi [1], Chandrasekhar et al. [3], and Chhillar et al.[4] discussed two-unit cold standby systems under different set of assumptions such as repair, replacement, inspection, etc. Wu and $\mathrm{Wu}[8]$ developed stochastic model for standby systems using concept of preventive maintenance after maximum operation time. Zhang and Wang [9] carried out availability analysis of cold standby system with constant failure rate. Some researchers, Osaki and Asakura [7], Gupta et al.[5] and Kumar and Saini [6] suggested some reliability models for cold standby redundant systems and single-unit systems in which all random variables are arbitrary distributed like Weibull distribution.

From the literature highlighted above, we find that a lot of research work is carried out for cold standby unit systems of identical units under the concepts of preventive maintenance and arbitrary distributions. But, the analysis of non-identical unit cold standby systems are not yet discussed by researchers under arbitrary distributions. For this, here an effort has been made to analyse the performance of a non-identical unit system having one original and one duplicate unit. A reliability model is developed by using concepts of preventive maintenance, repair, replacement and recurrence relations are derived with the help of regenerative point technique and semi-Markov processes for various reliability measures. A single repair facility has been provided to do repair and maintenance activities of original and duplicate unit. After a pre-specific time unit undergoes for preventive maintenance. Random variables are statistically independent and Weibulldistributed. The probability /cumulative density functions of direct transition time from regenerative state $i$ to a regenerative state $\mathrm{j}$ or to a failed state $\mathrm{j}$ visiting state $\mathrm{k}, \mathrm{r}$ once in $(0, t]$ have been denoted by $\mathrm{q}_{\mathrm{ij} . \mathrm{kr}}(\mathrm{t}) / \mathrm{Q}_{\mathrm{ij} . \mathrm{kr}}(\mathrm{t})$. The pdf of failure times of the original and duplicate unit are denoted by

$f(t)=\beta \eta t^{\eta-1} \exp \left(-\beta t^{\eta}\right)$ and

$f_{2}(t)=h \eta t^{\eta-1} \exp \left(-h t^{\eta}\right) \quad$ respectively. The probability density function of maximum operation time of original and duplicate unit is denoted by $g(t)=\alpha \eta t^{\eta-1} \exp \left(-\alpha t^{\eta}\right) . \quad$ The preventive maintenance rate of the original and duplicate units is denoted by the probability density function $g_{1}(t)=\gamma \eta t^{\eta-1} \exp \left(-\gamma t^{\eta}\right)$. The random variables corresponding to repair rate of the original and duplicate units have the probability density function $f_{1}(t)=k \eta t^{\eta-1} \exp \left(-k t^{\eta}\right)$ and

$f_{3}(t)=l \eta t^{\eta-1} \exp \left(-l t^{\eta}\right)$ respectively with $t \geq 0$ and $\theta, \eta, \alpha, \beta, h, k, l>0$. To improve the importance of the study, graphs are drawn for a particular case for mean time to system failure, availability and profit function. 


\begin{tabular}{|c|c|}
\hline Nomenclature & \\
\hline $\mathrm{O}$ & Operative unit \\
\hline DCs & Duplicate cold standby unit \\
\hline Do & Duplicative unit is operative \\
\hline$\sim / *$ & Symbol for Laplace -Steiltjes Transform (LST) / Laplace Transfor(LT) \\
\hline (S)/C) & Symbol for Laplace-Stieltjes convolution/Laplace convolution \\
\hline Fur/FUR & Denotes the failed original unit under repair/continuously under repair \\
\hline DFur/DFUR & Denotes the failed duplicate unit under repair/continuously under repair \\
\hline $\mathrm{DPm} / \mathrm{DPM}$ & $\begin{array}{l}\text { Denotes that duplicate unit under preventive maintenance/ continuously under preventive } \\
\text { maintenance }\end{array}$ \\
\hline $\mathrm{Pm} / \mathrm{PM}$ & Denotes that original unit under preventive maintenance/ continuously under preventive maintenance \\
\hline WPm/WPM & $\begin{array}{l}\text { Denotes that original unit waiting for preventive maintenance/ continuously waiting for preventive } \\
\text { maintenance }\end{array}$ \\
\hline DWPm/DWPM & $\begin{array}{l}\text { Denotes that duplicate unit waiting for preventive maintenance/ continuously waiting for preventive } \\
\text { maintenance }\end{array}$ \\
\hline Fwr/FWR & Original unit after failure waiting for repair/continuously waiting for repair \\
\hline DFwr / DFWR & Duplicate unit after failure waiting for repair/continuously waiting for repair \\
\hline MTSF & Mean Time to System Failure \\
\hline
\end{tabular}

\section{MODEL DESCRIPTION}

In this section, a stochastic model has been developed for two non-identical unit's systems using the concept of priority and arbitrary distributions. The system may be any of the following states describes as follows:
Out of these states $S_{0} S_{1} S_{2} S_{3}$ and $S_{4}$ are the operative and regenerative states while all other are non-regenerative and failed states.

3. TRANSITION PROBABILITIES AND





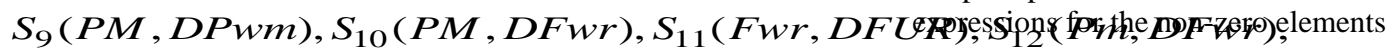

$$
p_{i j}=Q_{i j}(\infty)=\int q_{i j}(t) d t \text { As }
$$

$$
\begin{aligned}
& \mathrm{p}_{01}=\frac{\alpha}{\alpha+\beta}, \mathrm{p}_{02}=\frac{\beta}{\alpha+\beta}, \mathrm{p}_{10}=\frac{\gamma}{\alpha+h+\gamma}, \mathrm{p}_{1.10}=\frac{h}{\alpha+\gamma+h}=\mathrm{p}_{13.10}, \mathrm{p}_{19}=\frac{\alpha}{\alpha+\gamma+h}=\mathrm{p}_{14.9}, \\
& \mathrm{p}_{20}=\frac{k}{\alpha+k+h}, \mathrm{p}_{26}=\frac{h}{\alpha+h+k}=\mathrm{p}_{23.6}, \mathrm{p}_{27}=\frac{\alpha}{\alpha+h+k}=\mathrm{p}_{24.7}, \mathrm{p}_{30}=\frac{l}{l+\alpha+\beta}, \mathrm{p}_{40}=\frac{\gamma}{\alpha+\beta+\gamma},
\end{aligned}
$$$$
\mathrm{p}_{3.12}=\frac{\alpha}{\alpha+\beta+l}=\mathrm{p}_{33.12,} \mathrm{p}_{3.11}=\frac{\beta}{\alpha+\beta+l}=\mathrm{p}_{32.11}, \quad \mathrm{p}_{45}=\frac{\beta}{\alpha+\beta+\gamma}=\mathrm{p}_{42.5,} \mathrm{p}_{48}=\frac{\alpha}{\alpha+\beta+\gamma}=\mathrm{p}_{44.8},
$$

$\mathrm{p}_{52}=\mathrm{p}_{63}=\mathrm{p}_{74}=\mathrm{p}_{84}=\mathrm{p}_{94}=\mathrm{p}_{10.3}=\mathrm{p}_{11.2}=\mathrm{p}_{12.3}=1$ 
It can be easily verified that sum of all transition probabilities from each state is one.

\section{PERFORMANCE MEASURES: 4.1 Reliability and Mean Time to System Failure (MTSF)}

Let $\varphi_{i}(t)$ be the cdf of first passage time from the regenerative state $\mathrm{i}$ to a failed state. Regarding the failed state as absorbing state, we have the following recursive relations for

$\varphi_{i}(t)$ :

$\varphi_{i}(t)=\sum_{j} Q_{i, j}(t) \circledR \varphi_{j}(t)+\sum_{k} Q_{i, k}(t)$

Where $\mathrm{j}$ is an un-failed regenerative state to which the given regenerative state $i$ can transit and $k$ is a failed state to which the state $i$ can transit directly. Taking LST of above relation

(3) and solving for $\tilde{\phi}_{0}(s)$. The mean time to system failure

(MTSF) is given by MTSF $=\lim _{s \rightarrow 0} \frac{1-\tilde{\varphi}_{0}(s)}{s}$

$B_{i}^{R}(t)=W_{i}(t)+\sum_{j} q_{i, j}^{(n)}(t) \subset B_{j}^{R}(t), \quad B_{i}^{p m}(t)=W_{i}(t)+\sum_{j} q_{i, j}^{(n)}(t) \Subset B_{j}^{p m}(t)$

\subsection{Expected Number of Repairs, PM and Visits by Server}

regenerative state $i$ can transit through $\mathrm{n}$ transitions. By taking LT of (6) and solving for $B_{0}^{* R}(s)$ and $B_{0}^{* P m}(s)$. The busy period of the server due to repair and PM is given by $B_{0}^{R}=\lim _{s \rightarrow 0} s B_{0}^{* R}(s), B_{0}^{P m}=\lim _{s \rightarrow 0} s B_{0}^{* P m}(s)$

$E_{i}^{R}(t)=\sum_{j} Q_{i, j}^{(n)}(t) \circledast\left[\delta_{j}+E_{j}^{R}(t)\right], \quad E_{i}^{P m}(t)=\sum_{j} Q_{i, j}^{(n)}(t) \circledast\left[\delta_{j}+E_{j}^{P m}(t)\right]$

$N_{i}(t)=\sum_{j} Q_{i, j}^{(n)}(t) \circledR\left[\delta_{j}+N_{j}(t)\right]$

Let $E_{i}^{R}(t), E_{i}^{P m}(t)$ and $\mathrm{N}_{\mathrm{i}}(\mathrm{t})$ be the expected number of repairs $P M$ and visits by the server in $(0, t]$ given that the system entered the regenerative state $\mathrm{i}$ at $\mathrm{t}=0$. The recursive relations for these are given as

\subsection{Steady State Availability}

Let $\mathrm{A}_{\mathrm{i}}(\mathrm{t})$ be the probability that the system is in up-state at

$$
A_{i}(t)=M_{i}(t)+\sum_{j} q_{i, j}^{(n)}(t) \subseteq A_{j}(t)
$$

Where $j$ is any successive regenerative state to which the regenerative state $i$ can transit through $\mathrm{n}$ transitions. Taking

LT of above relations (4) and solving for $A_{0}^{*}(s)$. The steady state availability is given by
$A_{0}(\infty)=\lim _{s \rightarrow 0} s A_{0}^{*}(s)$

\subsection{Busy Period Analysis for Server}

$$
\text { Let } B_{i}^{R}(t) \text { and } B_{i}^{P m}(t) \text { be the probability that }
$$
the unit at an instant ' $\mathrm{t}$ ' given that the system entered state $\mathrm{i}$ at $\mathrm{t}=0$. The recursive relations for $\boldsymbol{B}_{i}^{R}(\boldsymbol{t})$ are as follows: the server is busy in repairing and preventive maintenance of
Where $\mathrm{j}$ is any regenerative state to which the given regenerative state $i$ transits and $\delta j=1$, if $j$ is the regenerative state where the server does job afresh, otherwise $\delta j=0$.

$$
R
$$

Taking LST of relations (7) and solving for $E_{0}(s)$. The expected numbers of repairs per unit time are given by $E_{0}^{R}(\infty)=\lim _{s \rightarrow 0} s E_{0}^{R}(s), \quad E_{0}^{P m}(\infty)=\lim _{s \rightarrow 0} s E_{0}^{P m}(s)$ and $N_{0}(\infty)=\lim _{s \rightarrow 0} s \tilde{N}_{0}(s)$

\subsection{Profit Analysis}

The profit incurred to the system model in steady state can be obtained as
$P=K_{0} A_{0}-K_{1} B_{0}^{P m}-K_{2} B_{0}^{R}-K_{3} E_{0}^{P m}-K_{4} E_{0}^{R}-K_{5} N_{0}$

(9)

$\mathrm{K}_{0}=$ Revenue per unit up-time of the system

$\mathrm{K}_{\mathrm{i}}=$ Cost per unit time for which server is busy due various repair activities 


\section{NUMERICAL RESULTS}

Table 1: MTSF vs. Failure Rate $(\beta)$ for shape parameter $\eta=0.5$

\begin{tabular}{|c|c|c|c|c|c|c|}
\hline$\beta$ & $\begin{array}{l}\alpha=2, \eta=0.5, \\
\gamma=5, \mathrm{k}=1.5, \\
\mathrm{~h}=0.009, \\
\mathrm{l}=1.4\end{array}$ & $\begin{array}{l}\alpha=2.4, \eta=0.5, \\
\gamma=5, \mathrm{k}=1.5, \\
\mathrm{~h}=0.009, \\
\mathrm{l}=1.4\end{array}$ & $\begin{array}{l}\alpha=2, \eta=0.5, \\
\gamma=7, \mathrm{k}=1.5, \\
\mathrm{~h}=0.009, \\
\mathrm{l}=1.4\end{array}$ & $\begin{array}{l}\alpha=2, \eta=0.5, \\
\gamma=5, \mathrm{k}=1.5, \\
\mathrm{~h}=0.01, \\
\mathrm{l}=1.4\end{array}$ & $\begin{array}{l}\alpha=2, \eta=0.5, \\
\gamma=5, \mathrm{k}=1.7, \\
\mathrm{~h}=0.009, \\
\mathrm{l}=1.4\end{array}$ & $\begin{array}{l}\alpha=2, \eta=0.5, \\
\gamma=5, \mathrm{k}=1.5, \\
\mathrm{~h}=0.009, \\
\mathrm{l}=2\end{array}$ \\
\hline 0.01 & 4.9918 & 2.1095 & 11.7910 & 4.9821 & 6.6949 & 4.9918 \\
\hline 0.02 & 4.7950 & 2.0642 & 10.7622 & 4.7861 & 6.3613 & 4.7950 \\
\hline 0.03 & 4.6123 & 2.0207 & 9.8940 & 4.6040 & 6.0577 & 4.6123 \\
\hline 0.04 & 4.4423 & 1.9788 & 9.1515 & 4.4345 & 5.7803 & 4.4423 \\
\hline 0.05 & 4.2836 & 1.9385 & 8.5094 & 4.2763 & 5.5260 & 4.2836 \\
\hline 0.06 & 4.1351 & 1.8998 & 7.9487 & 4.1283 & 5.2919 & 4.1351 \\
\hline 0.07 & 3.9960 & 1.8624 & 7.4548 & 3.9896 & 5.0758 & 3.9960 \\
\hline 0.08 & 3.8654 & 1.8264 & 7.0165 & 3.8594 & 4.8757 & 3.8654 \\
\hline 0.09 & 3.7426 & 1.7916 & 6.6251 & 3.7369 & 4.6898 & 3.7426 \\
\hline 0.1 & 3.6268 & 1.7581 & 6.2733 & 3.6214 & 4.5169 & 3.6268 \\
\hline
\end{tabular}

Table 2: MTSF vs. Failure Rate $(\beta)$ for shape parameter $\eta=1.0$

\begin{tabular}{|c|c|c|c|c|c|c|}
\hline$\beta$ & $\begin{array}{l}\alpha=2, \eta=1, \gamma=5, \mathrm{k} \\
=1.5, \mathrm{~h}=0.009, \\
\mathrm{l}=1.4\end{array}$ & $\begin{array}{l}\alpha=2.4, \eta=1, \gamma=5, \mathrm{k} \\
=1.5, \\
\mathrm{~h}=0.009, \\
\mathrm{l}=1.4\end{array}$ & $\begin{array}{l}\alpha=2, \eta=1, \\
\gamma=7, k=1.5, \\
h=0.009, \\
l=1.4\end{array}$ & $\begin{array}{l}\alpha=2, \eta=1, \\
\gamma=5, \mathrm{k}=1.5, \\
\mathrm{~h}=0.01, \\
\mathrm{l}=1.4\end{array}$ & $\begin{array}{l}\alpha=2, \eta=1, \\
\gamma=5, \mathrm{k}=1.7, \\
\mathrm{~h}=0.009, \\
\mathrm{l}=1.4\end{array}$ & $\begin{array}{l}\alpha=2, \eta=1, \\
\gamma=5, k=1.5, \\
h=0.009, \\
l=2\end{array}$ \\
\hline 0.01 & 5.9647 & 3.0460 & 13.8080 & 5.9531 & 8.0001 & 5.9647 \\
\hline 0.02 & 5.7599 & 2.9933 & 12.6725 & 5.7491 & 7.6420 & 5.7599 \\
\hline 0.03 & 5.5696 & 2.9426 & 11.7138 & 5.5594 & 7.3158 & 5.5696 \\
\hline 0.04 & 5.3921 & 2.8937 & 10.8934 & 5.3826 & 7.0175 & 5.3921 \\
\hline 0.05 & 5.2264 & 2.8466 & 10.1835 & 5.2174 & 6.7437 & 5.2264 \\
\hline 0.06 & 5.0712 & 2.8012 & 9.5632 & 5.0627 & 6.4914 & 5.0712 \\
\hline 0.07 & 4.9255 & 2.7573 & 9.0165 & 4.9176 & 6.2583 & 4.9255 \\
\hline 0.08 & 4.7886 & 2.7150 & 8.5310 & 4.7811 & 6.0422 & 4.7886 \\
\hline 0.09 & 4.6596 & 2.6741 & 8.0970 & 4.6525 & 5.8414 & 4.6596 \\
\hline 0.1 & 4.5379 & 2.6346 & 7.7067 & 4.5312 & 5.6542 & 4.5379 \\
\hline
\end{tabular}

Table 3: MTSF vs. Failure Rate $(\beta)$ for shape parameter $\eta=2.0$

\begin{tabular}{lllllll}
\hline$\beta$ & $\alpha=2, \eta=2$, & $\alpha=2.4, \eta=2, \gamma=5, \mathrm{k}$ & $\begin{array}{l}\alpha=2, \eta=2, \\
\gamma=7, \mathrm{k}=1.5,\end{array}$ & $\begin{array}{l}\alpha=2, \eta=2, \\
\gamma=5, \mathrm{k}=1.5,\end{array}$ & $\begin{array}{l}\alpha=2, \eta=2, \\
\gamma=5, \mathrm{k}=1.7,\end{array}$ & $\begin{array}{l}\alpha=2, \eta=2, \\
\mathrm{~h}=5, \mathrm{k}=1.5, \\
\mathrm{~h}=5, \mathrm{k}=1.5,\end{array}$ \\
$\mathrm{~h}=0.009$, & $\mathrm{h}=0.009$, & $\mathrm{h}=0.009$, & $\mathrm{h}=0.01$, & $\mathrm{l}=0.009$, \\
& $\mathrm{l}=1.4$ & $\mathrm{l}=1.4$ & $\mathrm{l}=1.4$ & $\mathrm{l}=1.4$ & $\mathrm{l}=1.4$ & $\mathrm{l}=2$ \\
\hline 0.01 & 8.9395 & 9.8394 & 20.8745 & 8.9222 & 11.9907 & 8.9395 \\
0.02 & 8.6486 & 9.5213 & 19.1946 & 8.6324 & 11.4757 & 8.6486 \\
0.03 & 8.3781 & 9.2255 & 17.7760 & 8.3630 & 11.0066 & 8.3781 \\
0.04 & 8.1260 & 8.9499 & 16.5621 & 8.1117 & 10.5776 & 8.1260 \\
0.05 & 7.8904 & 8.6923 & 15.5116 & 7.8770 & 10.1838 & 7.8904 \\
0.06 & 7.6698 & 8.4510 & 14.5936 & 7.6571 & 9.8209 & 7.6698 \\
0.07 & 7.4627 & 8.2247 & 13.7844 & 7.4507 & 9.4855 & 7.4627 \\
0.08 & 7.2680 & 8.0118 & 13.0659 & 7.2567 & 9.1746 & 7.2680 \\
0.09 & 7.0846 & 7.8113 & 12.4235 & 7.0739 & 8.8856 & 7.0846 \\
0.1 & 6.9116 & 7.6221 & 11.8458 & 6.9013 & 8.6162 & 6.9116 \\
\hline
\end{tabular}

Table 4: Availability vs. Failure Rate $(\beta)$ for shape parameter $\eta=0.5$

\begin{tabular}{lllllll}
\hline$\beta$ & $\alpha=2, \eta=0.5$, & $\alpha=2.4, \eta=0.5$, & $\alpha=2, \eta=0.5$, & $\alpha=2, \eta=0.5$, & $\alpha=2, \eta=0.5$, & $\alpha=2, \eta=0.5$, \\
& $\gamma=5, \mathrm{k}=1.5$, & $\gamma=5, \mathrm{k}=1.5$, & $\gamma=7, \mathrm{k}=1.5$, & $\gamma=5, \mathrm{k}=1.5$, & $\gamma=5, \mathrm{k}=1.7$, & $\gamma=5, \mathrm{k}=1.5$, \\
& $\mathrm{h}=0.009$, & $\mathrm{h}=0.009$, & $\mathrm{h}=0.009$, & $\mathrm{h}=0.01$, & $\mathrm{h}=0.009$, & $\mathrm{h}=0.009$, \\
& $\mathrm{l}=1.4$ & $\mathrm{l}=1.4$ & $\mathrm{l}=1.4$ & $\mathrm{l}=1.4$ & $\mathrm{l}=1.4$ & $\mathrm{l}=2$ \\
\hline 0.01 & 0.9405 & 0.9064 & 0.9734 & 0.9405 & 0.9417 & 0.9405 \\
0.02 & 0.9356 & 0.9005 & 0.9684 & 0.9356 & 0.9380 & 0.9357 \\
0.03 & 0.9307 & 0.8947 & 0.9634 & 0.9307 & 0.9343 & 0.9308 \\
0.04 & 0.9259 & 0.8889 & 0.9584 & 0.9259 & 0.9306 & 0.9260 \\
0.05 & 0.9211 & 0.8832 & 0.9535 & 0.9210 & 0.9269 & 0.9212 \\
0.06 & 0.9163 & 0.8776 & 0.9485 & 0.9162 & 0.9232 & 0.9164 \\
0.07 & 0.9115 & 0.8719 & 0.9436 & 0.9114 & 0.9195 & 0.9116 \\
\hline
\end{tabular}




\begin{tabular}{lllllll}
\hline 0.08 & 0.9067 & 0.8664 & 0.9387 & 0.9067 & 0.9159 & 0.9069 \\
0.09 & 0.9020 & 0.8608 & 0.9338 & 0.9019 & 0.9122 & 0.9021 \\
0.1 & 0.8973 & 0.8553 & 0.9290 & 0.8972 & 0.9085 & 0.8974 \\
\hline
\end{tabular}

Table 5: Availability vs. Failure Rate ( $\beta$ ) for shape parameter $\boldsymbol{\eta}=1.0$

\begin{tabular}{|c|c|c|c|c|c|c|}
\hline$\beta$ & $\begin{array}{l}\alpha=2, \eta=1, \gamma=5, \\
\mathrm{k}=1.5, \mathrm{~h}=0.00 \\
9, \\
\mathrm{l}=1.4\end{array}$ & $\begin{array}{l}\alpha=2.4, \eta=1, \gamma=5, \mathrm{k} \\
=1.5, \\
\mathrm{~h}=0.009, \\
\mathrm{l}=1.4\end{array}$ & $\begin{array}{l}\alpha=2, \eta=1, \\
\gamma=7, \mathrm{k}=1.5, \\
\mathrm{~h}=0.009, \\
\mathrm{l}=1.4\end{array}$ & $\begin{array}{l}\alpha=2, \eta=1, \\
\gamma=5, \mathrm{k}=1.5, \\
\mathrm{~h}=0.01, \\
\mathrm{l}=1.4\end{array}$ & $\begin{array}{l}\alpha=2, \eta=1, \\
\gamma=5, \mathrm{k}=1.7, \\
\mathrm{~h}=0.009, \\
\mathrm{l}=1.4\end{array}$ & $\begin{array}{l}\alpha=2, \eta=1, \\
\gamma=5, \mathrm{k}=1.5, \\
\mathrm{~h}=0.009, \\
\mathrm{l}=2\end{array}$ \\
\hline 0.01 & 0.8944 & 0.8623 & 0.9371 & 0.8943 & 0.8948 & 0.8945 \\
\hline 0.02 & 0.8919 & 0.8598 & 0.9343 & 0.8918 & 0.8927 & 0.8920 \\
\hline 0.03 & 0.8894 & 0.8573 & 0.9315 & 0.8893 & 0.8906 & 0.8895 \\
\hline 0.04 & 0.8869 & 0.8549 & 0.9288 & 0.8869 & 0.8885 & 0.8870 \\
\hline 0.05 & 0.8845 & 0.8525 & 0.9261 & 0.8844 & 0.8864 & 0.8846 \\
\hline 0.06 & 0.8821 & 0.8501 & 0.9234 & 0.8820 & 0.8844 & 0.8822 \\
\hline 0.07 & 0.8797 & 0.8477 & 0.9207 & 0.8796 & 0.8823 & 0.8798 \\
\hline 0.08 & 0.8773 & 0.8453 & 0.9180 & 0.8772 & 0.8803 & 0.8774 \\
\hline 0.09 & 0.8749 & 0.8430 & 0.9154 & 0.8749 & 0.8783 & 0.8750 \\
\hline 0.1 & 0.8726 & 0.8407 & 0.9128 & 0.8725 & 0.8763 & 0.8727 \\
\hline
\end{tabular}

Table 6: Availability vs. Failure Rate ( $\beta$ ) for shape parameter $\boldsymbol{\eta}=\mathbf{2 . 0}$

\begin{tabular}{|c|c|c|c|c|c|c|}
\hline$\beta$ & $\begin{array}{l}\alpha=2, \eta=2, \\
\gamma=5, \mathrm{k}=1.5, \\
\mathrm{~h}=0.009, \\
\mathrm{l}=1.4\end{array}$ & $\begin{array}{l}\alpha=2.4, \eta=2, \\
\gamma=5, k=1.5, \\
h=0.009, \\
l=1.4\end{array}$ & $\begin{array}{l}\alpha=2, \eta=2, \\
\gamma=7, k=1.5, \\
h=0.009, \\
l=1.4\end{array}$ & $\begin{array}{l}\alpha=2, \eta=2, \\
\gamma=5, \mathrm{k}=1.5, \\
\mathrm{~h}=0.01, \\
\mathrm{l}=1.4\end{array}$ & $\begin{array}{l}\alpha=2, \eta=2, \\
\gamma=5, \mathrm{k}=1.7, \\
\mathrm{~h}=0.009, \\
\mathrm{l}=1.4\end{array}$ & $\begin{array}{l}\alpha=2, \eta=2, \\
\gamma=5, \mathrm{k}=1.5, \\
\mathrm{~h}=0.009, \\
\mathrm{l}=2\end{array}$ \\
\hline 0.01 & 0.8715 & 0.8452 & 0.9109 & 0.8714 & 0.8717 & 0.8716 \\
\hline 0.02 & 0.8700 & 0.8438 & 0.9092 & 0.8700 & 0.8704 & 0.8701 \\
\hline 0.03 & 0.8686 & 0.8425 & 0.9076 & 0.8685 & 0.8691 & 0.8687 \\
\hline 0.04 & 0.8671 & 0.8411 & 0.9059 & 0.8670 & 0.8678 & 0.8672 \\
\hline 0.05 & 0.8657 & 0.8398 & 0.9043 & 0.8656 & 0.8666 & 0.8658 \\
\hline 0.06 & 0.8643 & 0.8385 & 0.9026 & 0.8642 & 0.8653 & 0.8644 \\
\hline 0.07 & 0.8629 & 0.8372 & 0.9010 & 0.8628 & 0.8641 & 0.8630 \\
\hline 0.08 & 0.8615 & 0.8359 & 0.8995 & 0.8614 & 0.8628 & 0.8616 \\
\hline 0.09 & 0.8601 & 0.8346 & 0.8979 & 0.8600 & 0.8616 & 0.8602 \\
\hline 0.1 & 0.8587 & 0.8333 & 0.8963 & 0.8586 & 0.8604 & 0.8588 \\
\hline
\end{tabular}

Table 7: Profit vs. Failure Rate $(\beta)$ for shape parameter $\boldsymbol{\eta}=0.5$

\begin{tabular}{|c|c|c|c|c|c|c|}
\hline$\beta$ & $\begin{array}{l}\alpha=2, \eta=0.5, \\
\gamma=5, k=1.5, \\
h=0.009, \\
l=1.4\end{array}$ & $\begin{array}{l}\alpha=2.4, \eta=0.5 \\
\gamma=5, \mathrm{k}=1.5, \\
\mathrm{~h}=0.009, \\
\mathrm{l}=1.4\end{array}$ & $\begin{array}{l}\alpha=2, \eta=0.5, \\
\gamma=7, \mathrm{k}=1.5, \\
\mathrm{~h}=0.009, \\
\mathrm{l}=1.4\end{array}$ & $\begin{array}{l}\alpha=2, \eta=0.5, \\
\gamma=5, \mathrm{k}=1.5, \\
\mathrm{~h}=0.01, \\
\mathrm{l}=1.4\end{array}$ & $\begin{array}{l}\alpha=2, \eta=0.5, \\
\gamma=5, \mathrm{k}=1.7, \\
\mathrm{~h}=0.009, \\
\mathrm{l}=1.4\end{array}$ & $\begin{array}{l}\alpha=2, \eta=0.5, \\
\gamma=5, k=1.5, \\
h=0.009, \\
l=2\end{array}$ \\
\hline 0.01 & 5932.3 & 6232.2 & 6156.2 & 5932.1 & 5940.0 & 5933.0 \\
\hline 0.02 & 5912.0 & 6203.7 & 6135.8 & 5911.8 & 5927.4 & 5912.8 \\
\hline 0.03 & 5891.7 & 6175.3 & 6115.5 & 5891.5 & 5914.7 & 5892.5 \\
\hline 0.04 & 5871.4 & 6147.1 & 6095.2 & 5.871 .2 & 5901.9 & 5872.3 \\
\hline 0.05 & 5851.2 & 6119.1 & 6074.8 & 5850.9 & 5889.1 & 5852.2 \\
\hline 0.06 & 5831.0 & 6091.3 & 6054.5 & 5830.7 & 5876.3 & 5832.0 \\
\hline 0.07 & 5810.8 & 6063.6 & 6.034 .2 & 5810.5 & 5863.5 & 5811.9 \\
\hline 0.08 & 5790.6 & 6036.1 & 6014.0 & 5790.3 & 5850.6 & 5791.8 \\
\hline 0.09 & 5770.5 & 6008.8 & 5993.7 & 5770.2 & 5837.7 & 5771.7 \\
\hline 0.1 & 5750.4 & 5981.6 & 5973.5 & 5750.1 & 5824.7 & 5751.7 \\
\hline
\end{tabular}

Table 8: Profit vs. Failure Rate $(\beta)$ for shape parameter $\eta=1.0$

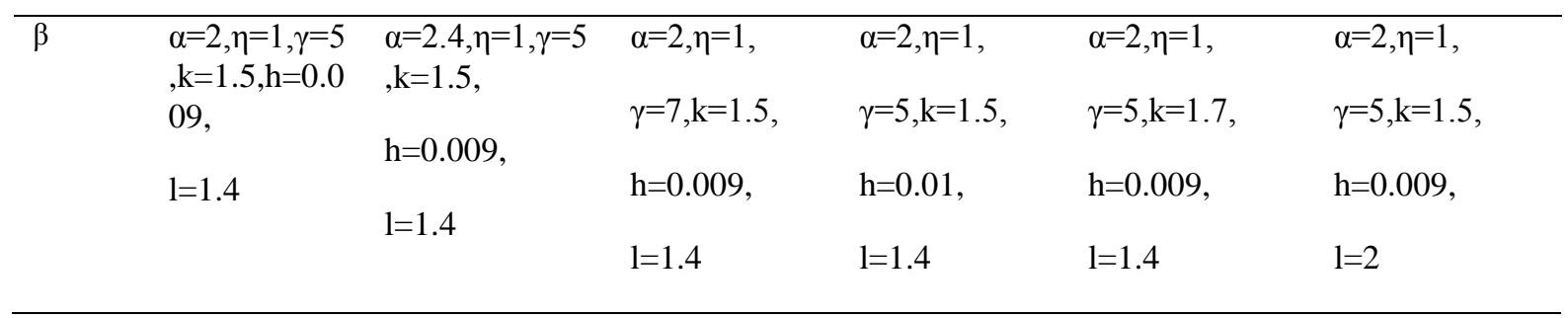




\begin{tabular}{lllllll}
\hline 0.01 & 5324.7 & 5326.4 & 5323.6 & 5640.6 & 5276.8 & 5323.9 \\
0.02 & 5312.1 & 5316.3 & 5310.9 & 5626.3 & 5263.8 & 5311.3 \\
0.03 & 5299.6 & 5306.2 & 5298.4 & 5612.1 & 5250.9 & 5298.8 \\
0.04 & 5287.2 & 5296.2 & 5286.0 & 5598.1 & 5238.1 & 5286.4 \\
0.05 & 5275.0 & 5286.3 & 5273.7 & 5584.2 & 5225.4 & 5274.1 \\
0.06 & 5262.8 & 5276.5 & 5261.5 & 5570.5 & 5.212 .9 & 5261.9 \\
0.07 & 5250.7 & 5266.7 & 5249.4 & 5.556 .8 & 5200.4 & 5249.8 \\
0.08 & 5238.8 & 5257.0 & 5237.4 & 5543.3 & 5188.0 & 5237.8 \\
0.09 & 5226.9 & 5247.4 & 5225.6 & 5530.0 & 5175.8 & 5226.0 \\
0.1 & 5215.1 & 5237.8 & 5213.8 & 5516.7 & 5163.6 & 5214.2 \\
\hline
\end{tabular}

Table 9: Profit vs. Failure Rate $(\boldsymbol{\beta})$ for shape parameter $\boldsymbol{\eta}=\mathbf{2 . 0}$

\begin{tabular}{lllllll}
\hline$\beta$ & $\alpha=2, \eta=2$, & $\alpha=2.4, \eta=2, \gamma=5, \mathrm{k}$ & $\alpha=2, \eta=2$, & $\alpha=2, \eta=2$, & $\alpha=2, \eta=2$, & $\alpha=2, \eta=2$, \\
& $\gamma=5, \mathrm{k}=1.5$, & $=1.5$, & $\gamma=7, \mathrm{k}=1.5$, & $\gamma=5, \mathrm{k}=1.5$, & $\gamma=5, \mathrm{k}=1.7$, & $\gamma=5, \mathrm{k}=1.5$, \\
& $\mathrm{h}=0.009$, & $\mathrm{h}=0.009$, & $\mathrm{h}=0.009$, & $\mathrm{h}=0.01$, & $\mathrm{h}=0.009$, & $\mathrm{h}=0.009$, \\
& $\mathrm{l}=1.4$ & $\mathrm{l}=1.4$ & $\mathrm{l}=1.4$ & $\mathrm{l}=1.4$ & $\mathrm{l}=1.4$ & $\mathrm{l}=2$ \\
\hline 0.01 & 4800.9 & 4687.7 & 5060.3 & 4800.5 & 4801.9 & 4801.6 \\
0.02 & 4792.9 & 4680.3 & 5050.9 & 4792.5 & 4795.0 & 4793.7 \\
0.03 & 4785.0 & 4673.0 & 5041.7 & 4784.6 & 4788.1 & 4785.8 \\
0.04 & 4777.1 & 4665.8 & 5032.5 & 4776.8 & 4781.3 & 4777.9 \\
0.05 & 4769.4 & 4658.6 & 5023.5 & 4769.0 & 4774.5 & 4770.2 \\
0.06 & 4761.7 & 4651.5 & 5014.6 & 4761.3 & 4767.8 & 4762.5 \\
0.07 & 4754.1 & 4644.4 & 5005.7 & 4753.7 & 4761.2 & 4754.9 \\
0.08 & 4746.6 & 4637.4 & 4997.0 & 4746.2 & 4754.7 & 4747.4 \\
0.09 & 4739.2 & 4630.4 & 4988.3 & 4738.8 & 4748.1 & 4740.0 \\
0.1 & 4731.8 & 4623.5 & 4979.8 & 4731.4 & 4741.7 & 4732.6 \\
\hline
\end{tabular}

\section{CONCLUSION}

In the section entitled numerical results, we obtained numerical values of performance measures such as mean time to system failure, availability and profit function for the proposed model with respect to failure rate $(\lambda)$ for various values of shape parameter $\eta=0.5,1,2$. For $\eta=1$, all random variables behaves as exponential distribution as a particular case of Weibull distribution while for $\eta=2$, it becomes Rayleigh. From, tables 1-9, we observe that the availability and profit of the system model decreases while MTSF increases with the increase of shape parameter. These measures shows a steep decline with the increase of failure rate of original and duplicate unit, maximum operation time whereas increase with respect to preventive maintenance of system and repair and replacement of original and duplicate unit. Finally, we conclude that by increasing the repair rate of the original and duplicate unit system can be made more profitable.

\section{REFERENCES}

[1] R. R. Agnihotri, S.K.Satsangi, Two Non-identical unit system with priority based repair and inspection, Microelectronic reliability, 36(2), 1996, 279-282.

[2] J. Cao, Y. Wu, Reliability analysis of a two-unit cold standby system with a Replaceable repair facility, Microelectronics Reliability, 29(2), 1989, 145-150.

[3] P. Chandrasekhar, R. Natarajan, V. S. S. Yadavalli, A study on a two unit standby System with Erlangian repair
Time, Asia-Pacific Journal of Operational Research, 21(03), 2004, 271-277.

[4] S. K. Chhillar, A. K. Barak, S. C. Malik, Reliability measures of a cold standby system with priority to repair over corrective maintenance subject to random shocks, International Journal of Statistics \& Economics $^{\mathrm{TM}}, 13(1), 2014$, 79-89.

[5] R. Gupta, P. Kumar, A. Gupta, Cost-benefit analysis of a two dissimilar unit cold standby system with Weibull failure and repair laws, Int. J. Syst. Assur. Eng. Manag., 4(4), 2013, 327-334.

[6] A. Kumar, M. Saini, Cost-benefit analysis of a singleunit system with preventive maintenance and Weibull distribution for failure and repair activities, Journal of Applied Mathematics, Statistics and Informatics, 10(2), 2014, 5-19.

[7] S. Osaki, T. Asakura, A two-unit standby redundant system with repair and preventive maintenance, Journal of Applied Probability, 7, 1970, 641-648.

[8] Q. Wu, S. Wu, Reliability analysis of two-unit cold standby repairable systems under Poisson shocks, Applied Mathematics and computation, 218(1), 2011, 171-182.

[9] Y. L. Zhang, G. J. Wang, A geometric process repair model for a repairable cold standby system with priority in use and repair, Reliability Engineering \& System Safety, 94(11), 2009, 1782-1787. 